\title{
STUDY OF TWO 3D FACE REPRESENTATION ALGORITHMS USING RANGE IMAGE AND CURVATURE-BASED REPRESENTATIONS
}

\author{
Agata Manolova, Krasimir Tonchev \\ Faculty of Telecommunications, Technical University of Sofia, 1000 Sofia, 8 Kl. Ohridski Str., Bulgria, \\ e-mail: amanolova@tu-sofia.bg, k_tonchev@tu-sofia.bg
}

\begin{abstract}
In this paper we present a comparative analysis of two algorithms for image representation with application to recognition of 3D face scans with the presence of facial expressions. We begin with processing of the input point cloud based on curvature analysis and range image representation to achieve a unique representation of the face features. Then, subspace projection using Principal Component Analysis (PCA) and Linear Discriminant Analysis (LDA) is performed. Finally classification with different classifiers will be performed over the 3D face scans dataset with 61 subject with 7 scans per subject (427 scans), namely two "frontal", one "look-up", one "look-down", one "smile", one "laugh", one "random expression". The experimental results show a high recognition rate for the chosen database. They demonstrate the effectiveness of the proposed 3D image representations and subspace projection for 3D face recognition. Copyright $(\mathbb{C}$ Research Institute for Intelligent Computer Systems, 2014. All rights reserved.
\end{abstract}

Keywords: 3D Face Recognition, Curvature Analysis, Range image representation, Principal Component Analysis, Linear Discriminant Analysis, Kernel Support Vector Machine classifier.

\section{INTRODUCTION}

Face recognition is a crucial part of many contemporary applications. Recent key applications in fields such as human-computer interface, identity verification, criminal face identification and surveillance systems need a reliable face recognition algorithms. Most of the approaches have focused on the use of 2D images but the decreasing cost of 3D acquisition systems and their increasing quality, together with the greater computational power available nowadays, will make real-time $3 \mathrm{D}$ systems for face recognition a commonplace in the near future.

According to [1] it appears that 2D face recognition techniques have exhausted their potential as they stumble on inherent problems of their modality such as differences in pose, lighting, expressions, and other characteristics that can vary between captures of a human face.

Recently, to overcome these challenges 3D facial recognition systems have been developed as a newly emerged biometric technique. It is showing promising results in terms of a high level of accuracy and reliability. Also it is more robust to face variation due to the different factors. A facebased biometric system consists of several subsystems: acquisition system performed by special devices (2D camera, 3D scanner or infrared camera), pre-processing unit, feature extraction unit, database and a recognition unit. In our scenario, an acquisition device may be a 3D-scanner that can record the facial information.

3D face recognition is a challenging task with a large number of proposed solutions [2,3]. With variations in pose and expression the identification of a face scan based on 3D geometry is difficult.

One common technique on 3D object recognition is based on the correspondence among scene points and model points in order to perform the recognition and to determinate the object pose and location [4]. Among the 3D free-form object descriptors to represent objects is the curvature of the local surface evaluated in each point, which is characterized by the directions in which the normal of the surface changes more and less quickly [5]. In [5], a set of twelve 3D feature extracted from segmented regions using curvature properties of the surface were experimented for face recognition using a database of 8 individual and 3 images per individual obtaining $95,5 \%$ recognition rate providing a previous $100 \%$ correct feature extraction.

Another popular approach to representing the face is the range image representation where the $3 \mathrm{D}$ point cloud is represented as a $2 \mathrm{D}$ image. The popularity of this approach is due to the many readily available methods for $2 \mathrm{D}$ facial recognition [6]. Also range images are robust to the change 
of color and illumination, which causes a significant problem in face recognition using 2D intensity images.

The rest of the paper is organized as follows: In the next section we present the technique for preprocessing of point cloud data, curvature analysis and range image representation of the 3D face image data. In Section 3 we present the approach for dimensionality reduction based on PCA and LDA. In that section we have described the classifiers for the subspace projections. In Section 4 we have presented the experimental results for the classification of the processed data with various classification techniques. Finally in section V we will present our future plans to develop these methods.

\section{PREPROCESSING OF THE POINT CLOUD DATA, RANGE IMAGE AND CURVATURE-BASED REPRESENTATION}

\subsection{POINT CLOUD FILTERING}

Compared to widely available CMOS and CCD technologies for capturing 2D images, the technology for 3D scanning is still immature in many aspects. There are multiple approaches to acquiring 3D data but all of them have drawbacks. The data acquired with 3D scanners contain undesired noise usually in the form of erroneous measurements or missing measurements. In the first case this noise appears as "spikes" in the data and in the second appears as "holes". An example is illustrated on Fig. 1.

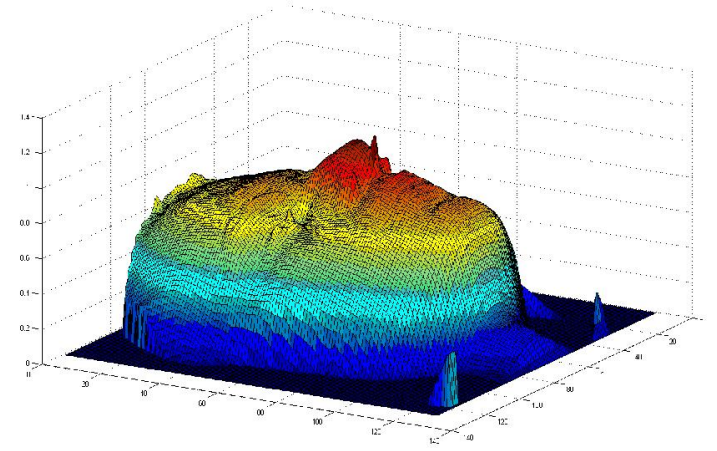

Fig. 1 - Example of spikes in the 3D scan under the nose and on the eye.

Usually samples are missing because the scanner fails to read the reflection of the laser beam at certain point, while the spike-like measurements are usually caused by reflection of wet surfaces such as the eyes [12]. The spikes exist because the laser beam is reflected by a glossy object such as wet skin areas. The holes on the other hand can be seen when the laser beam of the scanner is not reflected. Such situations can rise, for example, when the mouth of the person is open or the pupil of the eye is wide open. It may heavily influence the recognition processes so a preprocessing step is required.

To cope with these issues we apply preprocessing in three steps: face extraction, median filtration and smoothing. Median filtration is used to remove the spike-like measurements. The smoothing is performed using cubic interpolation. All missing points are filled by interpolation based on the closest points. Since the interpolation is applied in a least squares manner, a Gaussian noise is reduced also. Example of profile view of point cloud originated from scanner, filtered with median filter and the extracted face region is presented in Fig. 2.

The scanning process captures the face but also the region around the face including hair, neck, chests etc. These body parts do not contain information relevant to the face recognition process and need to be removed.
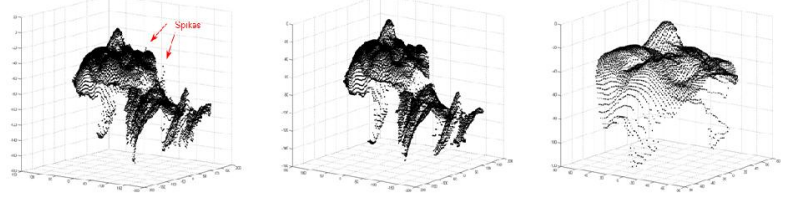

Fig. 2 - (from left to right): Example point cloud representation, filtered version of it and the extracted facial region.

To cope with these drawbacks we apply 3D median filtering of size $N_{\text {med }}$ elements. The steps to perform this filtration are as follows: first, for each point $\mathbf{x}_{i}=\left[x_{i}, y_{i}, z_{i}\right]^{T}$ of the point cloud data the nearest $N_{\text {med }}$ points are selected by calculating the Euclidean distance using only the $x$ and $y$ coordinates. Next the $z$ values of these $N_{\text {med }}$ points are arranged in increasing order and the value at index $\left\lfloor N_{\text {med }} / 2\right\rfloor+1$ is selected. This value represents the median value of the selected $N_{\text {med }}$ points, closest to $\mathbf{x}_{i}$. As a final step, the $z_{i}$ value of $\mathbf{x}_{i}$ is replaced with the median value.

This filtration removes the "spikes" and the "holes" and also noise induced by the scanner.

The next step in the preprocessing stage is face extraction (Fig. 1). This step is needed because the scanner captures data of body parts such as neck, shoulders and hair. This step can also be considered as face registration step because all faces are aligned to the same coordinate center. 


\subsection{RANGE IMAGE REPRESENTATION}

A common approach $[6,7]$ is to use all data points of the point cloud, located inside a sphere with radius $R_{F E}$ and center the tip of the nose. Using this assumption the point with maximum $z$ value is considered as the nose and translation of the $3 \mathrm{D}$ point cloud data is made such that this point is the center of $3 \mathrm{D}$ coordinate system. After the registration step all faces are aligned to a common coordinate system and are extracted such as to contain relevant and meaningful information only.

An example of range image representation and extracted face region is given on Fig 3. The intensity values of the range images represent inverse distance to the scanner i.e. the brighter the value the closer to the scanner.

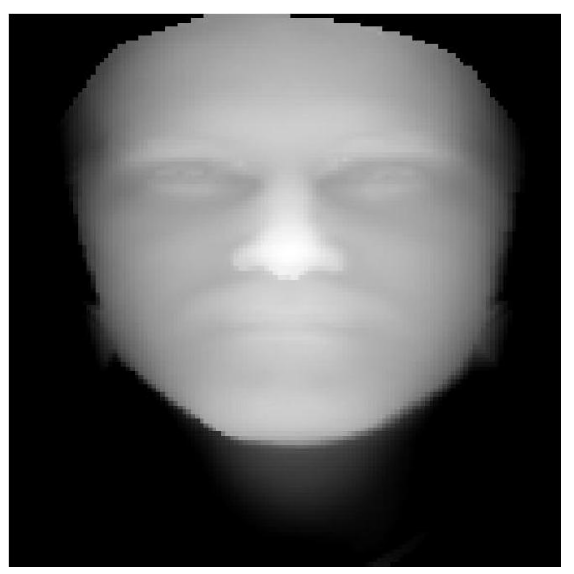

(a)

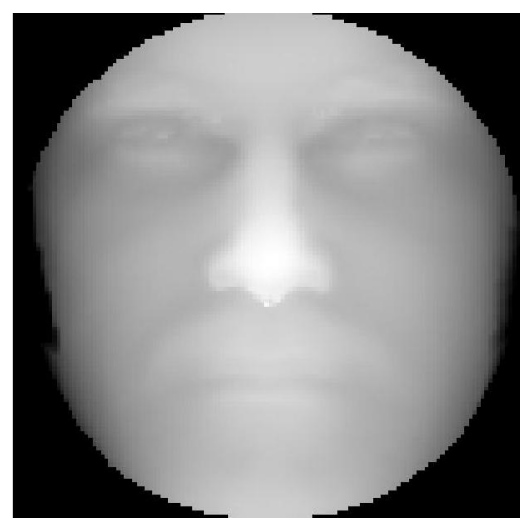

(b)

Fig. 3 - Range image representation of 3D point cloud data from scanner (a) and extracted face (b).

The range images are robust to the change of illumination and color because the value on each point represents the depth value which does not depend on illumination or color.

Also, range images are simple representations of $3 \mathrm{D}$ information. The $3 \mathrm{D}$ information in $3 \mathrm{D}$ mesh images is useful in face recognition, but its data is complex and difficult to handle. It is easy to utilize the $3 \mathrm{D}$ information of range images because the information of each point is explicit on a regularly spaced grid.

As a first step to range image representation the minimum and maximum values of the point cloud values are extracted:

$$
\begin{aligned}
& \operatorname{Min} X=\min \left(x_{i}\right), \operatorname{Max} X=\max \left(x_{i}\right) \\
& \operatorname{Min} Y=\min \left(y_{i}\right), \operatorname{Max} Y=\max \left(y_{i}\right) \\
& \operatorname{Min} Z=\min \left(z_{i}\right), \operatorname{Max} Z=\max \left(z_{i}\right)
\end{aligned}
$$

for each point $i=1, \ldots, N$, where $N$ is the number of points in the point cloud. Next a set of two regular grids, one for $x$ one for $y$ directions, are generated such that there are $N_{R I}$ equally spaced points between MinX and MaxX and MinY and MaxY respectively. These grids define the coordinate system of the range image. Next a linear interpolation is applied to the point cloud data such that the interpolated points lie on the previously defined grid. At the end rescaling of the $z$ values is applied such that $\operatorname{Min} Z=0$ and $\operatorname{Max} Z=1$. The result is $M$ by $M$ image with intensities in the range $[0,1]$.

\subsection{CURVATURE-BASED REPRESENTATION}

One of the main motivations for using curvature representation is their invariance to rotations. Besl and Jain [13] studied the 3D object recognition using range images. They calculated Gaussian curvature and mean curvature and used the signs of these surface curvatures to classify range image regions. Based on this 3D object recognition problem, Gordon devised a solution for a face recognition problem using range images [14].

Formally curvature represents the amount of local bending. The facial point cloud can be considered as 2D continuous surface parameterized over independent variables $u, v$.

Let $S$ be the facial surface in the form $z=f(x, y) \quad$ with parameterization $Z(u, v)=(u, v, f(u, v))$. The partial derivatives in the directions of the independent variables $Z_{u}, Z_{v}$ form a basis for the tangent plane at point $Z(u, v)$. Then the intersection of $S$ with a plane formed by the surface normal $n$ and a vector from the tangent plane is called normal section of $S$ at $(u, v)$ along the direction of the tangent vector. The curvature of the normal section is called normal curvature. Then the principal curvatures $k_{1}, k_{2},\left(k_{1} \geq k_{2}\right)$ are the 
maximum and the minimum of the normal curvatures at point $(u, v)$. Using the principal curvatures we calculate the mean $H$ and the Gaussian curvature $K$ by [8]:

$$
\begin{gathered}
K=k_{1} k_{2} \\
H=\frac{1}{2}\left(k_{1}+k_{2}\right)
\end{gathered}
$$

These values are calculated for each point of the $3 \mathrm{D}$ point cloud and they are the curvature based its representation. On fig. 4 an example of Gaussian and mean curvature representation of range image. Then the feature vector is formed by rearranging them as vectors and concatenation to form single vector.

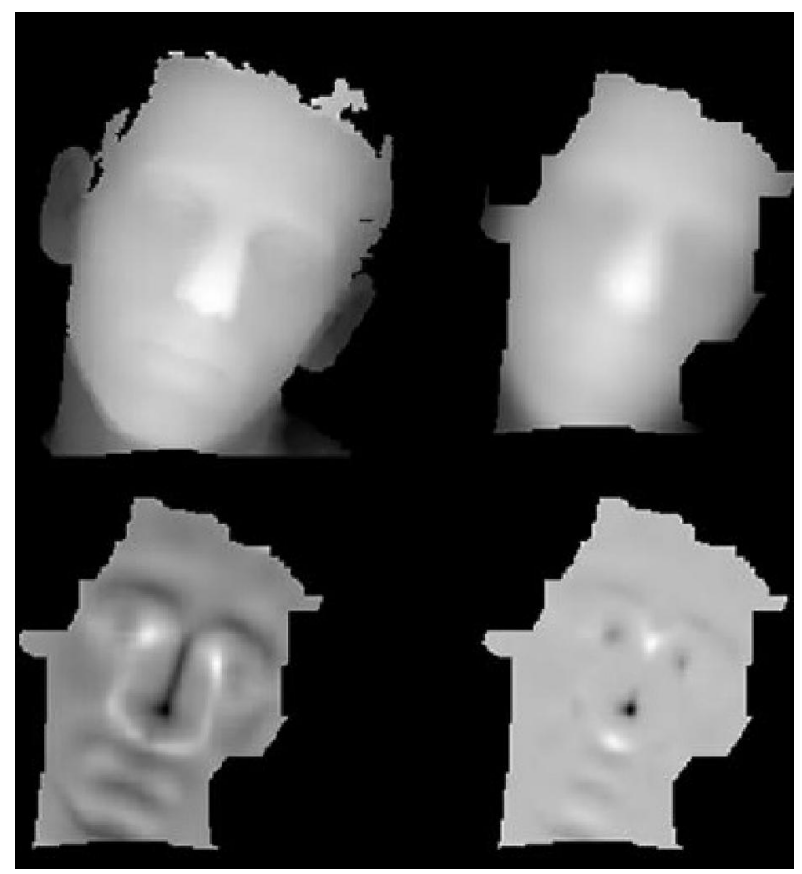

Fig. 4 - Top row, left to right: projected range image and its smoothed version. Second row: mean and Gaussian curvature map [21] (the darker zones are high curvature regions and lighter low curvature regions).

According to [15] this method shows that a great deal of information about facial features that cannot be seen from intensity images is contained in the curvature maps. This approach can deal with faces that differ in size, but needs extension to cope with changes in facial expression.

\section{SUBSPACE PROJECTION WITH PCA AND LDA}

For the subspace projection step we propose a combination of two very popular approaches for linear dimensionality reduction, namely Principal
Component Analysis (PCA) and Linear Discriminant Analysis (LDA).

\subsection{PRINCIPAL COMPONENT ANALYSIS}

The first approach is unsupervised technique for projection onto a subspace, trying to preserve most of the important information while reducing irrelevant noises.

From statistical point of view PCA seeks linear transformation of type $\mathbf{Y}=\mathbf{W}^{\mathrm{T}} \mathbf{X}$ which results in decorrelated output signals [9], where $\mathbf{X}$ is a matrix of the feature vectors. The major step in PCA is determining the basis $\mathbf{W}$. This basis is calculated by solving the problem of eigenvalues and eigenvectors of the covariance matrix of $\mathbf{X}$ :

$$
\mathbf{C}_{\mathbf{X}}=E\left\{(\mathbf{X}-\overline{\mathbf{X}})(\mathbf{X}-\overline{\mathbf{X}})^{\mathbf{T}}\right\}
$$

where $\overline{\mathbf{X}}$ is the mean value of the data. The solution of the eigenvalue problem is stated as:

$$
\mathbf{C}_{\mathbf{X}} \mathbf{W}=\mathbf{W} \Lambda
$$

Each of the columns of $\mathbf{W}$ represents a normalized eigenvector of $\mathbf{C}_{\mathbf{x}}$ and $\Lambda$ is diagonal matrix with the eigenvalues on the main diagonal. Dimensionality reduction can be achieved by first rearranging eigenvalues in descending order and then rearranging $\mathbf{W}$ to match $\Lambda$. Using the criteria Normalized Residual Mean Square Error (RMSE):

$$
\operatorname{RMSE}(b)=\sum_{i=1}^{b} \lambda_{i} / \sum_{i=1}^{p} \lambda_{i}>T
$$

where $T$ is a threshold representing the fraction of the power of the signal, the most relevant $b$ eigenvectors are selected.

Projecting the data on the PCA basis $\mathbf{W}$ has two advantages. First, this transformation preserves most of the important information and facilitates further calculations since the dimension of the projected data is much lower. Second, removing basis vectors of which the corresponding eigenvalues are lower is similar to filtering out non correlated noises. Thus PCA makes the process more robust to non informative variations.

\subsection{LINEAR DISCRIMINANT ANALYSIS}

The second technique is supervised and oriented towards classification. It seeks a subspace onto which the projected data can be separated by a hyperplane. 
Given $c$ classes $\omega_{i}, i=1, \ldots, c$ LDA seeks $c$ - 1 discriminating directions of type $y_{i}=\mathbf{w}_{i}^{\mathbf{T}} \mathbf{x}, i=1 \ldots c-1, \quad$ (or in matrix notation $\mathbf{y}=\mathbf{W}^{\mathbf{T}} \mathbf{x}$ ), where $\mathbf{x}$ is a PCA feature vector [9]. It is assumed that the dimensionality of the image space is $d \geq c$, which defines $\mathbf{W}$ as a rectangular matrix. Thus, LDA is a projection in a space with lower dimensionality.

The basis $\mathbf{W}$ is calculated by solving:

$$
J(\mathbf{W})=\underset{\mathbf{W}}{\arg \max } \frac{\left|\mathbf{W}^{\mathrm{T}} \mathbf{S}_{B} \mathbf{W}\right|}{\left|\mathbf{W}^{\mathrm{T}} \mathbf{S}_{W} \mathbf{W}\right|}
$$

$\mathbf{S}_{W}$ is called within-class scatter matrix representing the scatter of each class and $\mathbf{S}_{B}$ is between-class scatter matrix representing the scatter between the mean vectors of each class:

$$
\begin{aligned}
& \mathbf{S}_{W}=\sum_{i=1}^{c} \mathbf{S}_{i}, \text { where } \mathbf{S}_{i}=\sum_{\mathbf{x} \in \omega_{i}}\left(\mathbf{x}-\mathbf{m}_{i}\right)\left(\mathbf{x}-\mathbf{m}_{i}\right)^{\mathbf{T}} \\
& \mathbf{S}_{B}=\sum_{i=1}^{c} n_{i}\left(\mathbf{m}_{i}-\mathbf{m}\right)\left(\mathbf{m}_{i}-\mathbf{m}\right)^{\mathbf{T}}
\end{aligned}
$$

The vector $\mathbf{m}$ is the mean value of the data and $\mathbf{m}_{i}$ is the mean vector of the $i$-th class. The scalar $n_{i}$ defines the priors for $i$-th class. The matrix $\mathbf{S}_{B}$ is sum of $c$ matrices with rank one where at most $c-1$ of these matrices may be independent. Thus there exist not more than $c-1$ nonzero eigenvalues with their respective eigenvectors.

The solution of (8) is calculated by solving the generalized eigenvalue problem:

$\mathbf{S}_{B} \mathbf{w}_{i}=\lambda_{i} \mathbf{S}_{W} \mathbf{w}_{i}$

for each basis vector $\mathbf{w}_{i}, i=1, \ldots, c$, corresponding to the largest $c$ eigenvalues $\lambda_{i}, i=1, \ldots, c$.

Once the dimension of the data is reduced for the range image representation technique and the curvature-based representation using the feature vectors we apply multiple class classification using different classifiers to test the effectiveness of these two approaches.

\section{EXPERIMENTAL RESULTS}

We performed tests of the proposed approach using the "SHape REtrieval Contest 2008: 3D Face Scans" (SHREC) database [11]. All subjects in this database are Caucasian, scanned with Minolta Vi700 laser range scanner.

There are seven scans per subject, two are with neutral expression and the rest are with expression. Thus there are total 61 subject with 7 scans per subject (427 scans), namely two "frontal", one "look-up", one "look-down", one "smile", one "laugh", one "random expression".

SHREC scans are normalized for pose variations and a simple hole filling algorithm is applied. Moreover the tip of the nose is centered at the origin of the 3D coordinate system. This kind of normalization reduces the dependency of irrelevant variations and emphasize on the actual 3D face recognition. A sample of the database is presented on Fig. 5.

For both experiment we selected experimentally the radius of the sphere in the Face extraction step to be and the size of the median filter window to be $5 \times 5$.

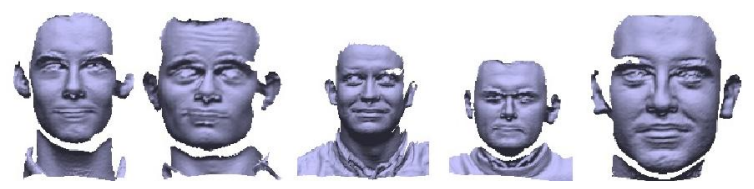

Fig. 5 - Sample from the 3D face scans of SHape REtrieval Contest 2008.

We performed two experiments on the point cloud face scans. In Experiment 1 we used the first two scans with frontal view (neutral expression) for training and the rest of the scans for testing. In Experiment 2 we used 2 frontal scans, one scan with smiling expression and one "random expression" scan for training and the rest for testing.

For the experiments we used Matlab Pattern Recognition Toolbox PRTools 4.2.4 (http://prtools.org/) [16] and the LIBSVM package (http://www.csie.ntu.edu.tw/ cjlin/libsvm/) [17] for the kernel Support Vector Classifier.

For our classification task we use several standard classifiers.

We apply nearest neighbors classifier (KNN) for classification of unknown point cloud into one of the available classes. This classifier is relatively simple technique which assigns the class label of the unknown data based on the closest training samples.

Closeness is defined by the distance between the samples and the class label assignment is made by selecting the class label of the majority of the closest KNN samples. In our approach we use the advantages of the Euclidean measure to compute the distance between samples and we tested the approach by varying the number of neighbors between 1, 3 and 5. Only the best results for the recognition rate are presented [22]. 
Minimum least square linear classifier (Fisher classifier), normal densities based quadratic (multiclass) classifier (QDC) and Naïve Bayes classifier are also used in the experiment.

The normal based quadratic classifier assumes that the classes are described by multi-normal distributions each with its own covariance matrix. If the covariance matrices are singular we use a regularized version of this classifier by enlarging the diagonal values of the matrix [20].

The Fisher linear discriminant is obtained maximizing the ratio of between-scatter to withinscatter - the Fisher criterion, which for two classes is basically the normal based linear classifier. If the covariance matrix is singular we use a regularized version of this classifier by enlarging the diagonal values of the matrix [20].

The naive Bayes classifier has several properties that make it useful in practice. One of the most important is the separation of the class conditional feature distributions which means that each distribution can be independently estimated as a one dimensional distribution. This helps solve the problem of the curse of dimensionality, such as the need for datasets that scale exponentially with the number of features [19].

We also applied kernel-based SVM. Each kernel defines an implicit transformation from objective space into a usually higher dimensional feature space. We applied kernel Support Vector Machines with the pure distance substitution linear kernel $\left(k_{l i n}\right)$ and the Gaussian radial basis kernel $\left(k_{r b f}\right)$. Depending on the chosen kernel, the geometry of this induced feature space can be very specific. Using RBF kernels for example, the points in feature space all lie on a hyper sphere around the origin with a radius one [18]. One of the main characteristics of kernel-based SVM is that their runtime and space complexity is basically independent of the dimensionality of the input space, but rather scales with the number of data points used for training. In our case we use only a fraction of the dataset for training.

The values of the penalty error $C$ and the parameter for the Gaussian radial basis kernel are logarithmically varying along a suitable grid and only the best recognition rates are presented. Because this is multiclass recognition problem we used the "one versus all" procedure for the Support Vector Machines where classification is done by a max-wins voting strategy.

For the size of the range images we selected $N_{R I}=64$, thus the range images space is of size 4096. We preserve $95 \%$ of the energy of the PCA eigenvalues which results in 107 eigenvectors, i.e. 107 dimensional feature spaces.
The results for the range image representation in terms of recognition rate in $\%$ are presented in Table 1.

Table 1. Experimental results for the range image representation in terms of recognition rate in $\%$.

\begin{tabular}{|l|c|c|}
\hline \multicolumn{1}{|c|}{ Classifier } & $\begin{array}{c}\text { Recognition Rate } \\
\text { Experiment 1 }\end{array}$ & $\begin{array}{c}\text { Recognition Rate } \\
\text { Experiment 2 }\end{array}$ \\
\hline Fisher classifier & 71,93 & 77,56 \\
\hline QDC & 81.64 & 89,43 \\
\hline Naïve Bayes & 78,88 & 86.24 \\
\hline KNN & 91.78 & 95.45 \\
\hline SVM $k_{\text {lin }}$ & 64.92 & 75,93 \\
\hline SVM $k_{r b f}$ & 93.36 & 96.64 \\
\hline
\end{tabular}

On Fig. 6 is represented the training set for Experiment 2 in function of the first two features to demonstrate the highly nonlinear data representation.

For the curvature-based representation after preserving $95 \%$ of the energy of the eigenvectors i.e. $\mathrm{T}=0.95$ the resultant PCA basis is with 112 eigenvectors.

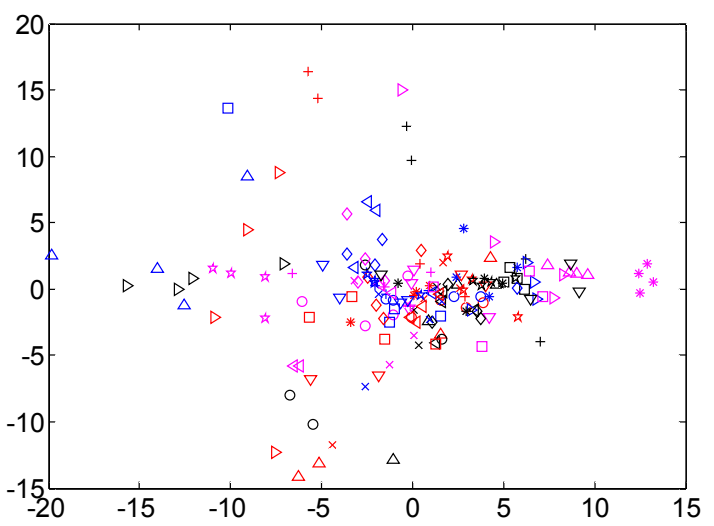

Fig. 6 - The training set for Experiment 2 in function of the first two features.

The results for the curvature-based image representation in terms of recognition rate in $\%$ are presented in Table 2 below.

Table 2. Experimental results for the curvature-based image representation in terms of recognition rate in $\%$.

\begin{tabular}{|l|c|c|}
\hline Classifier & $\begin{array}{c}\text { Recognition Rate } \\
\text { Experiment 1 }\end{array}$ & $\begin{array}{c}\text { Recognition Rate } \\
\text { Experiment 2 }\end{array}$ \\
\hline $\begin{array}{l}\text { Fisher } \\
\text { classifier }\end{array}$ & 63.93 & 69.22 \\
\hline QDC & 76.38 & 83.65 \\
\hline Naïve Bayes & 72.68 & 79.22 \\
\hline KNN & 78.78 & 85.16 \\
\hline SVM $k_{\text {lin }}$ & 69.56 & 74.87 \\
\hline SVM $k_{r b f}$ & 87.12 & 91.56 \\
\hline
\end{tabular}




\section{CONCLUSION}

In this paper we have presented comparative analysis to 3D Face recognition techniques based on range image and curvature-based image representations.

The 3D range image representation technique results in easy to handle data because the value on each point represents the depth information. The range images are robust to the change of illumination and color.

The curvature-based representation is robust to faces that differ in size, but needs extension to cope with changes in facial expression as the results for the recognition rate show.

The experimental results show a high recognition rate for the SHREC 3D Face database. These experiments demonstrate the effectiveness of the proposed 3D image representations and subspace projection for 3D face recognition. The recognition is successful with most classifiers but the best classifier performance in the two representations is the SVM Gaussian radial basis kernel. The linear classifiers such as Fisher and SVM $k_{\text {lin }}$ perform less well than the other nonlinear classifiers, this is due to the highly nonlinear data representation.

In the future additional tests will be performed adding more classification techniques to try and confirm the method's robustness against additive noise. Also different test will be included with different number of retained feature vectors. There is a need of improving the curvature-based representation to take into account the changes in facial expression.

\section{ACKNOWLEDGMENT}

This work was sponsored by the Ministry of Education and Science of Bulgaria, National Foundation "Science and Research", SlovenianBulgarian R\&D joint project, NSF Grant DNTS/Slovenia 01/08 - "Fast and Reliable 3D Face Recognition".

\section{REFERENCES}

[1] G. Eason, B. Noble, and I. N. Sneddon, Threedimensional face recognition in the presence of facial expressions: an annotated deformable model approach, Journal of IEEE Transactions on Pattern Analysis and Machine Intelligence, (29) 4 (April 2007), pp. 640-649.

[2] K. Bowyer, K. Chang, P. Flynn, A survey of approaches and challenges in 3D and multimodal 3D $+2 \mathrm{D}$ face recognition, Computer Vision and Image Understanding, (101) 1 (2006), pp. 1-15.
[3] A. Scheenstra, A. Ruifrok, R. C. Veltkamp, A survey of $3 \mathrm{D}$ face recognition methods, in AVBPA, 2005, pp. 891-899.

[4] R. Campbel, P. Flynn, A survey of free-form object representation and recognition techniques, Computer Vision and Image Understanding, (81) (2001), pp. 166-210.

[5] P. Hallinan, G. Gordon, A. Yuille, P. Giblin, D. Mumford, Two and Three-dimensional patterns of the face, Ed. A. K. Peters, 1999.

[6] B. Gökberk, H. Dutağacı, A. Ulaş, L. Akarun, B. Sankur, Representation plurality and fusion for 3D face recognition, IEEE Transactions on Systems, Man, and Cybernetics-Part B: Cybernetics, (38) 1 (2008), pp. 155-173.

[7] A. Mian, M. Bennamoun, R. Owens, An efficient multimodal 2D-3D hybrid approach to automatic face recognition, IEEE Transactions on Pattern Analysis and Machine Intelligence, (29) 11 (2007), pp. 1927-1943.

[8] H. Tanaka, M. Ikeda, H. Chiaki, Curvaturebased face surface recognition using spherical correlation, Principal directions for curved object recognition, Proceedings of the Third IEEE International Conference on Automatic Face and Gesture Recognition, 1998, pp. 372377, 1998.

[9] K. Fukunaga, Statistical Pattern Recognition, Academic Press, NY, 1989.

[10] G. Shakhnarovish, T. Darrell, P. Indyk, ed., Nearest-Neighbor Methods in Learning and Vision, MIT Press, 2005.

[11] F. Haar, M. Daoudi, R. Veltkamp, SHape REtrieval contest 2008: 3D face scans, Proceedings of the IEEE International Conference on Shape Modeling and Applications, 2008, pp. 225-226.

[12] A.B. Moreno, A. Sanchez, J.F. Vlez, F.J. Daz, Face recognition using 3D surface-extracted descriptors, Proceedings of the Irish Machine Vision and Image Processing Conference, 2003.

[13] P. Besl and R. Jain, Invariant surface characteristics for 3D object recognition in range images, Computer Vision, Graphics and Image Processing, (33) (1986), pp. 33-80.

[14] G. Gordon, Face recognition based on depth maps and surface curvature, Geometric Methods in Computer Vision, (SPIE 1570) (July 1991), pp. 1-12.

[15] J. Lee, 3D face recognition using range images, literature survey report, Department of Electrical and Computer Engineering, The University of Texas, Austin, 2005.

[16] F. van der Heijden, R. P.W. Duin, D. de Ridder and D. M.J. Tax, Classification, parameter estimation and state estimation, an engineering 
approach using Matlab, John Wiley \& Sons, 2004.

[17] C.-C. Chang and C.-J. Lin. LIBSVM: a library for support vector machines, $A C M$ Transactions on Intelligent Systems and Technology, (2) 3 (2011), pp. 1-27.

[18] M. Eigensatz, Insights into the Geometry of the Gaussian Kernel and an Application in Geometric Modeling, Master thesis, Swiss Federal Institute of Technology Zurich, March 13, 2006

[19] Harry Zhang, The optimality of naive bayes, Proceedings of the Conference FLAIRS, Miami Beach, Florida, USA, 2004, pp. 562-567.

[20] E. Pękalska and R.P.W. Duin, Classifiers for dissimilarity-based pattern recognition, in: A. Sanfeliu, J.J. Villanueva, M. Vanrell, R. Alquezar, A.K. Jain, J. Kittler (eds.), Proceedings of the $15^{\text {th }}$ International Conference on Pattern Recognition ICPR15, Barcelona, Spain, September 3-7, 2000.

[21] A. Colombo, C. Cusano, R. Schettini, 3D face detection using curvature analysis, Pattern Recognition, (39) 3 (March 2006), pp. 444 455.

[22] K. Tonchev, A. Manolova, I. Paliy, Comparative Analysis of 3D Face Recognition Algorithms Using Range Image and CurvatureBased Representations, The 7th IEEE International Conference on Intelligent Data Acquisition and Advanced Computing Systems: Technology and Applications (IDAACS'2013), 12-14 September 2013, Berlin, Germany, Vol. 1, pp. 394-398.

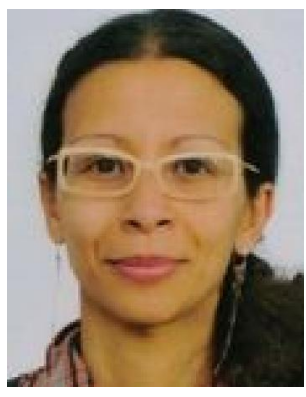

Ass. Prof. Agata Manolova, is the Assistant Professor with the Faculty of Telecommunications at the Technical University of Sofia (TU-Sofia), Bulgaria. She finished her PhD conjointly between the TU-Sofia and the University of Grenoble, France. Her domains of interest are Pattern Recognition, Computer Vision, Statistics, Image and Video processing. Dr. Manolova has participated in several scientific projects both national and international concerning object and human action recognition in video sequences, compression of multispectral and hyper spectral images, developing an online educational system for the students at the TU-Sofia. In 20132014 she was invited scholar with a Fulbright Visiting Scholar grant in the Computer Science Department at University of Arkansas at Little Rock, USA.

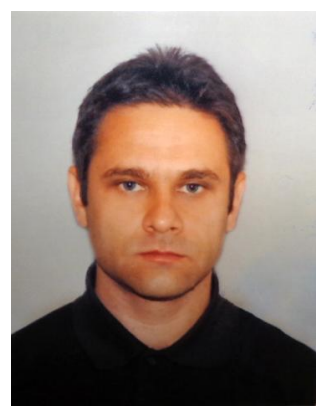

Krasimir Tonchev, is a $P h D$ student at the Department of Radio communications and Video Technologies, Faculty of Telecommunications, technical University of Sofia, Bulgaria. His main interests are Kernelbased Support Vector Machines, age and gender recognition, $3 D$ face models, computer vision. He has participated in several scientific projects both national and international for the development of reliable face recognition methods. With collaboration with other authors he has published several papers in conferences such as WSEAS, IDAACS and others. 